\title{
Combined noninvasive language mapping by navigated transcranial magnetic stimulation and functional MRI and its comparison with direct cortical stimulation
}

\author{
Sebastian Ille, MD, ,,2 Nico Sollmann, MD, ${ }^{1,2}$ Theresa Hauck, ${ }^{1,2}$ Stefanie Maurer, ${ }^{1,2}$ \\ Noriko Tanigawa, MAs, ${ }^{5}$ Thomas Obermueller, MD, ${ }^{1,2}$ Chiara Negwer, MD, $, 1,2$ Doris Droese ${ }^{4}$ \\ Claus Zimmer, MD, ${ }^{2,3}$ Bernhard Meyer, MD, ${ }^{1}$ Florian Ringel, MD,1 and Sandro M. Krieg, MD ${ }^{1,2}$ \\ 1Department of Neurosurgery; ${ }^{2}$ TUM-Neuroimaging Center; ${ }^{3}$ Section of Neuroradiology, Department of Radiology; and \\ ${ }^{4}$ Department of Anesthesiology, Klinikum rechts der Isar, Technische Universität München, Munich, Germany; and ${ }^{5}$ Faculty of \\ Linguistics, Philology, \& Phonetics, University of Oxford, United Kingdom
}

\begin{abstract}
OBJECT Repetitive navigated transcranial magnetic stimulation (rTMS) is now increasingly used for preoperative language mapping in patients with lesions in language-related areas of the brain. Yet its correlation with intraoperative direct cortical stimulation (DCS) has to be improved. To increase rTMS's specificity and positive predictive value, the authors aim to provide thresholds for rTMS's positive language areas. Moreover, they propose a protocol for combining rTMS with functional MRI (fMRI) to combine the strength of both methods.

METHODS The authors performed multimodal language mapping in 35 patients with left-sided perisylvian lesions by using rTMS, fMRI, and DCS. The rTMS mappings were conducted with a picture-to-trigger interval (PTI, time between stimulus presentation and stimulation onset) of either 0 or $300 \mathrm{msec}$. The error rates (ERs; that is, the number of errors per number of stimulations) were calculated for each region of the cortical parcellation system (CPS). Subsequently, the rTMS mappings were analyzed through different error rate thresholds (ERT; that is, the ER at which a CPS region was defined as language positive in terms of rTMS), and the 2-out-of-3 rule (a stimulation site was defined as language positive in terms of rTMS if at least 2 out of 3 stimulations caused an error). As a second step, the authors combined the results of fMRI and rTMS in a predefined protocol of combined noninvasive mapping. To validate this noninvasive protocol, they correlated its results to DCS during awake surgery.
\end{abstract}

RESULTS The analysis by different rTMS ERTs obtained the highest correlation regarding sensitivity and a low rate of false positives for the ERTs of $15 \%, 20 \%, 25 \%$, and the 2-out-of-3 rule. However, when comparing the combined fMRI and rTMS results with DCS, the authors observed an overall specificity of $83 \%$, a positive predictive value of $51 \%$, a sensitivity of $98 \%$, and a negative predictive value of $95 \%$.

CONCLUSIONS In comparison with FMRI, rTMS is a more sensitive but less specific tool for preoperative language mapping than DCS. Moreover, rTMS is most reliable when using ERTs of 15\%,20\%, 25\%, or the 2-out-of-3 rule and a PTI of 0 msec. Furthermore, the combination of fMRI and rTMS leads to a higher correlation to DCS than both techniques alone, and the presented protocols for combined noninvasive language mapping might play a supportive role in the language-mapping assessment prior to the gold-standard intraoperative DCS.

http://thejns.org/doi/abs/10.3171/2014.9.JNS14929

KEY WORDS language; tumor; transcranial magnetic stimulation; functional magnetic resonance imaging; awake surgery; diagnostic and operative techniques

$\mathrm{T}$ HE resection of tumors within or adjacent to language-eloquent brain regions is still a neurosurgical quest, and a profound presurgical workup is crucial to achieving the best functional and oncological re- sult. ${ }^{6,71}$ Today, the most precise way to localize individual language-eloquent regions is direct cortical stimulation (DCS) during awake craniotomy. ${ }^{9}, 12,26,44,45,54,62,65,74$ Using only DCS, however, we cannot provide the longitudinal

ABBREVIATIONS BOLD = blood oxygen level-dependent; CPS = cortical parcellation system; DCS direct cortical stimulation; ER = error rate; ERT error rate threshold; $\mathrm{fMRI}=$ functional MRI; IPI = interpicture interval; NPV = negative predictive value; $n T M S$ navigated TMS; $P P V=$ positive predictive value; $P T I=$ picture-to-trigger interval; RMT = resting motor threshold; ROC = receiver operating characteristic; rTMS repetitive navigated TMS; TMS = transcranial magnetic stimulation.

SUBMITTED April 25, 2014. ACCEPTED September 11, 2014.

INCLUDE WHEN CITING Published online March 6, 2015; DOI: 10.3171/2014.9.JNS14929.

DISCLOSURE The study was completely financed by institutional grants of the Department of Neurosurgery and the Section of Neuroradiology, TU Munich. S.K. is consultant for BrainLAB AG (Feldkirchen, Germany). The other authors declare that they have no conflict of interest affecting this study. 
noninvasive follow-up examinations that might enable us to include plastic reshaping of cortical language function in our oncological considerations. . $^{11,16,17,20,42,54,76}$

Although we are gaining more and more information about the distribution of human language function and the associated networks, mapping is still very complex and has to be further refined..$^{10,16,30,31,46,54,65}$ Thus, there is a need to assess functional cortical organization of language function by noninvasive methods. Navigated transcranial magnetic stimulation (nTMS) is one of these noninvasive techniques. Regarding the mapping of motor function, nTMS has already demonstrated its usefulness for clinical practice. ${ }^{22,33,35,52,53,77}$ Furthermore, repetitive navigated TMS (rTMS) and nonnavigated TMS are able to localize cortical language function. ${ }^{18,19,40,48,69,80}$ Its clinical applicability and correlation to DCS during awake surgery have repeatedly been shown as well. ${ }^{36,37,51,68,75}$ Although rTMS language mapping has already experienced some improvement, ${ }^{40}$ the standard for preoperative, noninvasive language mapping remains functional MRI (fMRI). ${ }^{21}$ Yet this technique, though well established, has failed to provide reliable preoperative language mapping, showing only minor correlation with intraoperative DCS. ${ }^{24,57,82} \mathrm{An}-$ other study regarding the localization of language and motor areas by fMRI has confirmed the selection of a more aggressive therapeutic approach for the use of preoperative fMRI. ${ }^{49}$ On the other hand, a review of studies for the preoperative mapping of language function by fMRI has concluded that the effect on surgical planning is to be the only approved clinical use and that even this technique has to be further investigated. ${ }^{4}$

Thus, this study has been designed to investigate how the results of rTMS language mapping have to be analyzed to find the highest correlation with DCS, and to refine this promising and, in this field, aspiring, technique so as to play a supportive role toward a multimodal approach in the future. Moreover, and with the same purpose, we have evaluated the data to create a protocol for noninvasive language mapping through the combination of rTMS and fMRI and correlated the results to intraoperative DCS as well.

\section{Methods \\ Ethics Approval}

The experimental setup was approved by the local ethics committee of our university in accordance with the Declaration of Helsinki. All patients provided written informed consent for this study before rTMS.

\section{Study Design}

The study was designed to be prospective and nonrandomized.

\section{Patients}

The study was conducted in 35 consecutive patients (22 males and 13 females) with left-sided perisylvian brain lesions. Inclusion criteria were the presence of a left-sided perisylvian brain lesion, planned awake craniotomy, age of at least 18 years, and signed informed consent. Exclusion criteria were general TMS exclusion criteria, such as pacemaker or cochlear implant, ${ }^{56}$ as well as severe aphasia and age younger than 18 years.
All patients were scheduled for awake craniotomy in our neurosurgical department between April 2011 and January 2014, and all underwent preoperative language mapping by rTMS. Additionally, 27 patients (17 males and 10 females) were preoperatively examined by fMRI using an object-naming paradigm. Moreover, aphasia grading adapted from the Aachener Aphasia Test was done 3 times: before surgery, the 5th day after surgery, and 3 months after surgery. ${ }^{28}$ All lesions were located in the left-hemispheric perisylvian brain regions, and 32 patients were right-handed. Table 1 gives an overview on the patient cohort, including age, aphasia, and tumor location. To some extent, the data of some of these patients were previously part of earlier studies..$^{36,51}$

\section{Navigational MRI Scan}

All patients underwent navigational MRI scanning in a 3-T MR scanner (Achieva 3T, Philips Medical System) using an 8-channel phased array head coil. The protocol contained a 3D gradient echo sequence (TR 9 msec, TE 4 $\mathrm{msec}, 1-\mathrm{mm}^{3}$ isovoxel covering the whole head, 6-minuteand-58-second acquisition time) and an intravenous contrast administration of $0.1 \mathrm{mmol} / \mathrm{kg}$ body weight gadopentetate dimeglumine (Magnograf, Marotrast $\mathrm{GmbH}$ ) for anatomical coregistration. This 3D data set was then used for the preoperative rTMS language mapping and for the intraoperative neuronavigation..$^{51,67}$

Besides these sequences, the scanning protocol included a T2-weighted FLAIR sequence (TR 12,000 msec, TE $140 \mathrm{msec}$, TI $2500 \mathrm{msec}, 30$ slices with a 1-mm gap, voxel size $0.9 \times 0.9 \times 4 \mathrm{~mm}, 3$-minute acquisition time).

\section{Preoperative fMRI Language Mapping}

For blood oxygen level-dependent (BOLD) fMRI, each subject underwent an fMRI object-naming task. The sequence parameters were as follows. For fMRI, echo planar imaging was performed with the following parameters: train length $43 \mathrm{msec}$, TR $2500 \mathrm{msec}$, and TE $35 \mathrm{msec}$. Within 2 minutes and 53 seconds, 64 dynamic sets were acquired, each consisting of 32 contiguous axial 4-mm slices with an in-plane resolution of $2.75 \times 2.75 \mathrm{~mm}$. Parallel imaging (SENSE) was used to diminish susceptibilityrelated artifacts (SENSE factor 2).

After the examination, the fMRI data were transferred to an external workstation (Extended MR Workspace, Philips Medical Systems) and were postprocessed by the IViewBOLD package blinded to the rTMS results. After motion correction and spatial smoothing (2D gaussian filter with 4-mm full width at half maximum, kernel $2 \times$ 2 pixel) statistical parametric maps were generated using the general linear model. We chose a hemodynamic delay of $2 \times$ TR, a single predictor, and a t-value threshold of 2.5 . Only clusters with positive correlation, larger than 40 voxels in size, were considered to be activated areas. The validity of the results was checked by review of the timeintensity diagrams of the activated voxels, as also previously described..$^{34,68}$

\section{Preoperative rTMS Language Mapping \\ Experimental Setup}

The rTMS language mapping was performed using 
TABLE 1. Patient characteristics

\begin{tabular}{|c|c|c|c|c|c|c|c|c|c|}
\hline \multirow{2}{*}{$\begin{array}{c}\text { Case } \\
\text { No. }\end{array}$} & \multirow[b]{2}{*}{ Age (yrs) } & \multirow[b]{2}{*}{ Tumor Type } & \multirow[b]{2}{*}{ Lesion Location* } & \multicolumn{3}{|c|}{ Mapping Techniques } & \multicolumn{3}{|c|}{ Aphasia Gradingt } \\
\hline & & & & DCS & fMRI & rTMS & Preop & 5th POD & 3rd PON \\
\hline 1 & 25 & C & $\operatorname{trIFG}$ & Yes & Yes & Yes & 0 & 0 & 0 \\
\hline 2 & 28 & $\mathrm{AA}$ & anG & Yes & Yes & Yes & 0 & $1 \mathrm{~A}$ & 0 \\
\hline 3 & 62 & GBM & opIFG & Yes & Yes & Yes & 0 & 0 & 0 \\
\hline 4 & 56 & AA & mMTG & Yes & Yes & Yes & 0 & 3B & $2 A$ \\
\hline 5 & 53 & $\mathrm{AA}$ & pMTG & Yes & Yes & Yes & 0 & 0 & 0 \\
\hline 6 & 43 & GBM & oplFG & Yes & Yes & Yes & $1 \mathrm{~A}$ & $1 \mathrm{~A}$ & $1 \mathrm{~A}$ \\
\hline 7 & 51 & GBM & anG & Yes & Yes & Yes & $2 B$ & $2 B$ & $2 B$ \\
\hline 8 & 50 & GBM & anG & Yes & Yes & Yes & $2 \mathrm{~A}$ & $3 A$ & $1 \mathrm{~A}$ \\
\hline 9 & 51 & GBM & vPrG & Yes & Yes & Yes & $1 \mathrm{~A}$ & $2 \mathrm{~A}$ & 0 \\
\hline 10 & 40 & GBM & pSTG & Yes & Yes & Yes & $2 B$ & 0 & 0 \\
\hline 11 & 34 & C & mMFG & Yes & Yes & Yes & 0 & 0 & 0 \\
\hline 12 & 63 & $\mathrm{DA}$ & pSTG & Yes & Yes & Yes & 1B & $2 B$ & 1B \\
\hline 13 & 47 & GBM & pMTG & Yes & Yes & Yes & $2 B$ & $2 B$ & $2 B$ \\
\hline 14 & 56 & GBM & pMTG & Yes & Yes & Yes & 0 & $2 A$ & 0 \\
\hline 15 & 47 & $\mathrm{AA}$ & aSMG & Yes & Yes & Yes & 1B & $2 B$ & 0 \\
\hline 16 & 33 & GBM & mSTG & Yes & No & Yes & 0 & $3 A$ & 0 \\
\hline 17 & 53 & GBM & oplFG & Yes & Yes & Yes & $1 \mathrm{~A}$ & $2 A$ & $1 \mathrm{~A}$ \\
\hline 18 & 32 & C & anG & Yes & No & Yes & $3 \mathrm{~A}$ & 0 & 0 \\
\hline 19 & 47 & GBM & oplFG & Yes & Yes & Yes & 0 & 0 & 0 \\
\hline 20 & 52 & GBM & oplFG & Yes & No & Yes & $2 A$ & $2 \mathrm{~A}$ & $2 A$ \\
\hline 21 & 43 & DA & oplFG & Yes & Yes & Yes & 0 & $2 \mathrm{~A}$ & 0 \\
\hline 22 & 30 & AA & anG & Yes & Yes & Yes & $1 \mathrm{~A}$ & $1 \mathrm{~A}$ & 0 \\
\hline 23 & 48 & GBM & oplFG & Yes & Yes & Yes & 0 & $2 \mathrm{~A}$ & $1 \mathrm{~A}$ \\
\hline 24 & 74 & GBM & aSTG & Yes & Yes & Yes & $2 A$ & $2 A$ & - \\
\hline 25 & 41 & AA & pSTG & Yes & Yes & Yes & $2 B$ & 1B & 1B \\
\hline 26 & 47 & GBM & anG & Yes & Yes & Yes & $1 \mathrm{~A}$ & 0 & 0 \\
\hline 27 & 49 & DA & oplFG & Yes & Yes & Yes & 0 & 1B & 0 \\
\hline 28 & 27 & AVM & mSTG & Yes & Yes & Yes & 0 & $1 \mathrm{~A}$ & 0 \\
\hline 29 & 66 & $\mathrm{DA}$ & oplFG & Yes & No & Yes & 0 & $1 \mathrm{~A}$ & 0 \\
\hline 30 & 38 & AA & opIFG & Yes & No & Yes & 0 & $1 \mathrm{~A}$ & 0 \\
\hline 31 & 33 & $\mathrm{OA}$ & $\operatorname{trlFG}$ & Yes & No & Yes & 0 & 0 & 0 \\
\hline 32 & 31 & GNT & vPrG & Yes & No & Yes & 0 & 0 & 0 \\
\hline 33 & 51 & GBM & vPrG & Yes & Yes & Yes & $2 A$ & $1 \mathrm{~A}$ & $1 \mathrm{~A}$ \\
\hline 34 & 24 & $\mathrm{DA}$ & $\mathrm{mPrG}$ & Yes & Yes & Yes & 0 & 0 & - \\
\hline 35 & 27 & GBM & anG & Yes & No & Yes & 0 & 1B & - \\
\hline \multicolumn{10}{|c|}{$\begin{array}{l}\text { AA = anaplastic astrocytoma (WHO Grade III); AVM = arteriovenous malformation; C = cavernoma; DA = diffuse astrocytoma (WHO Grade II); } \\
\text { GBM = glioblastoma (WHO Grade IV); GNT = glioneural tumor (WHO Grade I); OA = oligoastrocytoma (WHO Grade III); POD = postoperative } \\
\text { day; POM = postoperative month; - = not performed. } \\
\text { * Refer to Table } 2 \text { for the definitions of lesion locations. } \\
\text { † Aphasia grading: } 0 \text { = no aphasia; } 1=\text { mild aphasia; } 2 \text { = moderate aphasia; } 3 \text { = severe aphasia; } A=\text { predominantly motor impairment; } B= \\
\text { predominantly sensory impairment. }\end{array}$} \\
\hline
\end{tabular}

nTMS eXimia NBS version 3.2.2 and Nexstim NBS 4.3 with a NEXSPEECH module (Nexstim Oy), as described earlier. ${ }^{51,68,75}$ First, the 3D T1-weighted MRI scan of each patient and the patient's head were coregistered. The stimulated brain area during the examination was visualized using a stereotactic camera and reflectors fastened to the patient's head with an elastic strap to track the coil position. ${ }^{51}$ The induced electric field in the brain was visualized over the $3 \mathrm{D}$ reconstruction, and the intracranial stimulation points were saved for later examination. . $^{29,58,59}$ After the coregistration, the individual patient's resting motor threshold (RMT) was defined by motor mapping of the cortical representation of the contralateral abductor pollicis brevis muscle, as published before. ${ }^{34}$ This RMT was then used as a basic value for the rTMS mapping procedure, using an object-naming task, consisting of 131 colored pictures of common objects. ${ }^{40,51,68}$ The pictures were displayed with an interpicture interval (IPI) of 
2.5 seconds. As described earlier, the individual mapping frequency and intensity was defined using our standard protocol: $48,67,68$

1) RMT in the left hemisphere was determined thoroughly.

2) A train of 5-7 rTMS bursts was administered to the ventral precentral gyrus and the opercular inferior frontal gyrus:

a) $5 \mathrm{~Hz}, 5$ pulses, $100 \% \mathrm{RMT}$;

b) $7 \mathrm{~Hz}, 5$ pulses, $100 \% \mathrm{RMT}$;

c) $7 \mathrm{~Hz}, 7$ pulses, $100 \%$ RMT.

3) The setup $(a-c)$ that caused the most language errors was identified by the patient's and examiner's impressions and in unclear cases supported by video analysis.

4) If there was no clear difference in the effect on language, the most comfortable frequency was chosen.

5) If naming was not interrupted clearly by rTMS, the intensity was increased to $110 \%-120 \%$ RMT, and Step 1 was repeated.

6) If significant pain was reported, the stimulation intensity was lowered to $80 \%-90 \%$ RMT to avoid any discomfort that might interfere with the consecutive-response evaluation..$^{19}$ This was also done if $100 \%$ RMT was painful.

The display time (the time the pictures were presented on the screen) was $700 \mathrm{msec}$. Another parameter for the variation of rTMS language mapping is the picture-to-trigger interval (PTI). The PTI describes the time between the presentation of the stimulus on the screen and the onset of the rTMS burst. Twenty-five patients were examined with a PTI of $300 \mathrm{msec}$, and 10 patients were examined with a PTI of $0 \mathrm{msec}$. There is evidence in earlier studies for the justification of both PTIs. ${ }^{30,55,63,81}$ Thus, our protocol was modified after 25 patients.

\section{TMS Language-Mapping Procedure}

Before rTMS language mapping, baseline recording was performed twice without stimulation to adapt the picture data set to the individual vocabulary. The patients had to name the presented pictures in their mother tongue as quickly and precisely as possible, and the number of baseline errors was documented for each patient. Misnamed pictures were discarded. The remaining pictures were presented time locked to a train of rTMS pulses, and the stimulation coil was randomly moved in between the visual display of 2 images. To achieve maximum field induction, the coil was placed perpendicular to the skull, ${ }^{40}$ and 80-120 sites were stimulated 3 times each with a distance of approximately $10 \mathrm{~mm}$. The minimum cortical field strength of the induced electric field was $55 \mathrm{~V} / \mathrm{m}$.

For later detailed and objective analyses, the baseline performance and the stimulation trials were video recorded. ${ }^{40}$

\section{TMS Data Analysis}

First, the videorecorded rTMS language mappings were analyzed in comparison with the baseline performance. The detected language errors were documented and categorized into 7 groups: no responses, performance errors, hesitations, neologisms, semantic paraphasias, phonologic paraphasias, and circumlocutions. ${ }^{9,67}$ Language errors related to muscle stimulations or pain were discarded.
To ensure that the evaluation was performed objectively, we analyzed the mappings blinded to the stimulation sites or the tumor location. ${ }^{37,40,51}$ After the video analysis, the detected language errors sorted by error type were assigned to the cortical parcellation system (CPS) as published by Corina et al. ${ }^{8}$ Figure $1 \mathrm{~A}$ shows the CPS, including the abbreviations of the mapped cortical regions. The abbreviations are further explained in Table 2. The next step was to calculate the error rates (ERs). The ERs were calculated for each area of the CPS, defined by the number of errors per number of stimulations. ${ }^{36,51}$ Moreover, each area of the CPS was analyzed regarding the 2-outof-3 rule. As already mentioned, each stimulation site was stimulated 3 times. A CPS region was defined as language positive in terms of the 2-out-of-3 rule if at least 2 out of 3 stimulations caused a predefined language error. ${ }^{26,65}$

Subsequently, the rTMS raw data were analyzed with 12 error rate thresholds (ERTs). The ERT is defined as the ER at which a CPS region has been defined as language positive in terms of TMS. The 12 ERTs were determined ongoing from $0 \%$, in $5 \%$ steps, to $50 \%$ of stimulations (ERTs $>0 \%, \geq 5 \%, \geq 10 \%, \geq 15 \%, \geq 20 \%, \geq 25 \%, \geq 30 \%$, $\geq 35 \%, \geq 40 \%, \geq 45 \%, \geq 50 \%$ ) and the 2-out-of- 3 rule.

\section{Language Mapping During Awake Craniotomy \\ Setup}

A mixture of bupivacaine and epinephrine was used for local anesthesia of the galea and dura. By continuous infusion of remifentanil and propofol, an adequate level of anesthesia and sedation were maintained. The patient's head was fixed in a Mayfield clamp, the reflector for navigation was attached to it, and a neuronavigation system (Vectorvision Sky or Brainlab Curve, Brainlab AG) was used to locate the surgical tools and the cortical stimulation electrode based on the same 3D MRI used during the rTMS session.

Ten minutes before language mapping, analgesia and sedation were discontinued. Regarding the wakefulness, a Ramsay sedation score of 2 (patient awake, calm, and cooperative) was targeted for the language-mapping procedure. After completion of cortical mapping, the operation was continued under conscious sedation. ${ }^{50}$

\section{Language-Mapping Procedure}

The cortical stimulation was performed using a bipolar stimulation electrode (distance of $5 \mathrm{~mm}$, Inomed Medizintechnik $\mathrm{GmbH}$ ). The stimulation intensity was between 0 and $20 \mathrm{~mA}$, with a frequency of $50 \mathrm{~Hz}$ and duration of 4 seconds. The stimulation sites were placed 5-10 mm apart, and a surface electroencephalogram with a bandpass filter of $10 \mathrm{~Hz}$ to $1.5 \mathrm{kHz}$ was recorded to detect epileptic seizures. For the intraoperative mapping by DCS and the preoperative mapping by rTMS the same pictures were used, with the difference of starting the object naming during the operation with the matrix sentence, "This is a ...". 32 The cortical sites were stimulated 3 times, and a site was considered language positive if at least 2 out of 3 stimulations led to a language error. Thus the 2-out-of-3 rule was used. The positive sites were marked with letters and were transferred to the navigation system using the navigation pointer. ${ }^{44,50,51}$ 


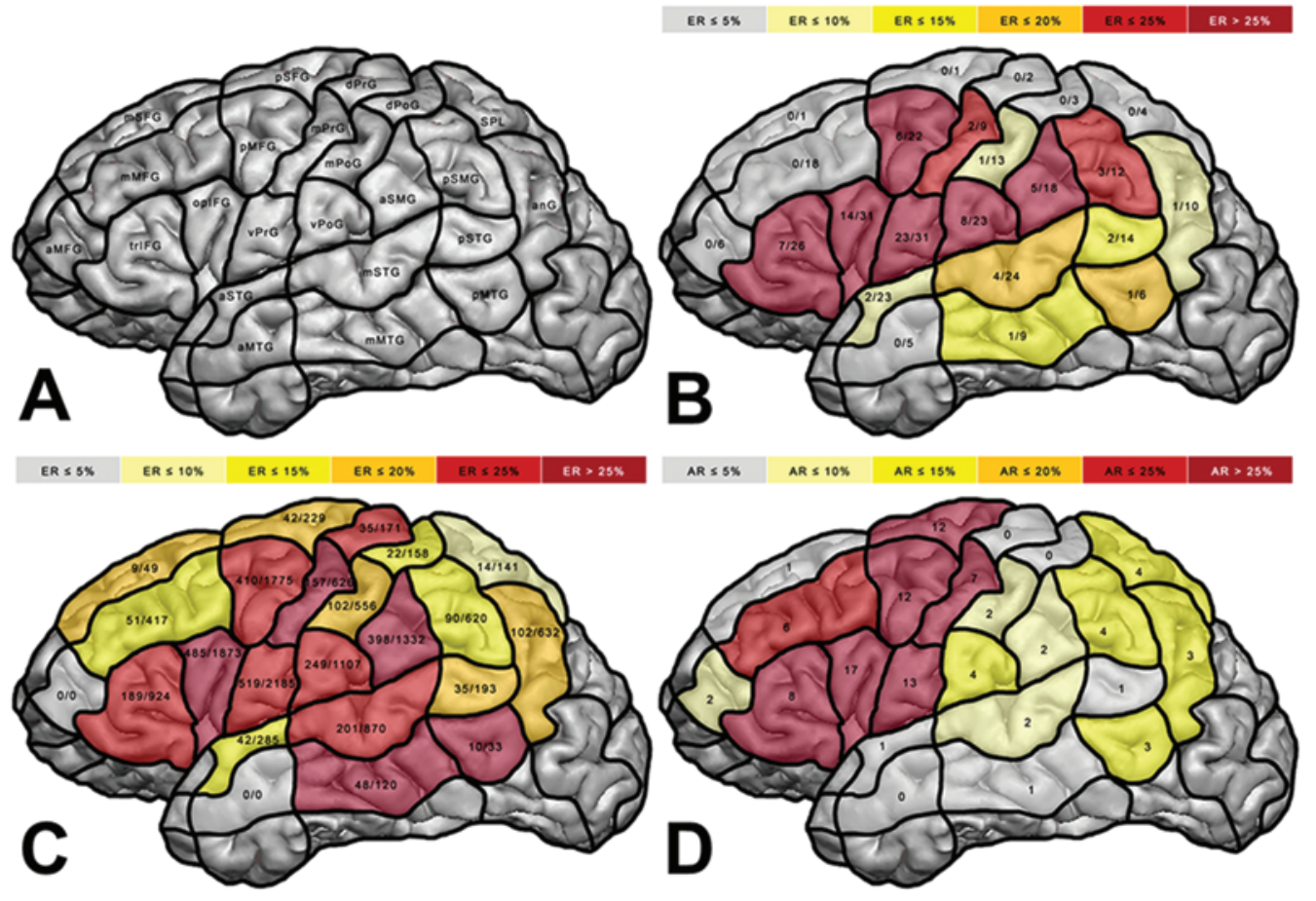

FIG. 1. CPS and total number of errors per number of stimulations and activations of each method. A: The CPS as described by the study of Corina et al., ${ }^{8}$ including the abbreviations of the mapped regions. The abbreviations are explained in Table 2. B and C: For DCS $(B)$ and rTMS $(C)$ language mapping we illustrate the total number of errors per number of stimulations for each CPS region, highlighted by the respective color of the associated ER. D: For fMRI language mapping, we demonstrate the total number of positive BOLD signals. The related activation rate is calculated by the total number of positive BOLD signals per number of patients who performed fMRI language mapping.

\section{Data Analysis}

\section{Anatomical Localization}

The 37 regions of the CPS as defined by Corina et al. were used to compare the results of the different techniques of language mapping and to provide sufficient statistical data to compare the methods (Fig. 1A) ${ }^{8}$ First, the positive and negative language sites of the intraoperative mapping by DCS were assigned to the CPS. The stimulated sites, then transferred to the neuronavigation system by the navigation pointer, could be located exactly in a 3D environment for further analysis.

After the video analysis, the rTMS-induced language errors were matched with the associated stimulation sites on the 3D MRI. This was separately done for the 12 ERT groups (0\% ERT to 50\% ERT in 5\% steps and by the 2-out-of-3 rule).

The positive language sites examined by fMRI with an object-naming task were anatomically located through the coronal, sagittal, and axial slices, which were fused with the BOLD signal. They were assigned to the CPS as well. To compare the overall results of rTMS and DCS with fMRI language mapping, we calculated an activation rate by the total number of positive BOLD signals per number of patients who underwent fMRI language mapping $(\mathrm{n}=$ 27).

\section{Stimulation Assessment for the Comparison of rTMS and DCS}

Representing the gold standard, the results of DCS's intraoperative language mappings provided the ground truth for every comparison. For the comparison of rTMS and DCS language mapping, the results of both methods were assigned to the CPS separately for each patient. Then the raw data for calculating the receiver operating characteristic (ROC) curves were created as follows: if a CPS region gave rise to language positivity during DCS and rTMS mapping (according to the chosen ERT), the region was documented as a true positive for this patient. If both mappings indicated this region to be language negative, it was documented as a true negative. When rTMS mapping led to an ER above the respective ERT in the corresponding CPS region but the DCS mapping did not, the CPS region was defined as a false positive for this patient. Moreover, a CPS region was documented as a false negative when the region's DCS mapping led to language errors but the ER was below the ERT during rTMS mapping.

For the definition of language positivity and negativity in terms of rTMS, we used the different ERTs. For a better understanding, we provide some examples. For the analysis with an ERT of 0\%, a CPS region was counted as language positive in terms of rTMS if any stimulation of this region elicited a language error. For the comparison of DCS to rTMS with an ERT of 5\%, the CPS regions were taken as language positive only if the ER was at least equal to $5 \%$. In the same way, the ROCs were also calculated up to an ERT of 50\%. Moreover, we used the 2-outof-3 rule as an ERT.

Ten rTMS language mappings were performed with a PTI of 0 msec, and 25 patients were mapped with a PTI 
TABLE 2. Abbreviations of the anatomical cortical areas according to the CPS

\begin{tabular}{cl}
\hline Abbreviation & \multicolumn{1}{c}{ Anatomy } \\
\hline aMFG & Anterior middle frontal gyrus \\
\hline aMTG & Anterior middle temporal gyrus \\
\hline anG & Angular gyrus \\
\hline aSMG & Anterior supramarginal gyrus \\
\hline aSTG & Anterior superior temporal gyrus \\
\hline dPoG & Dorsal postcentral gyrus \\
\hline dPrG & Dorsal precentral gyrus \\
\hline MMFG & Middle middle frontal gyrus \\
\hline MMTG & Middle middle temporal gyrus \\
\hline mPoG & Middle postcentral gyrus \\
\hline mPrG & Middle precentral gyrus \\
\hline mSFG & Middle superior frontal gyrus \\
\hline mSTG & Middle superior temporal gyrus \\
\hline opIFG & Opercular inferior frontal gyrus \\
\hline pMFG & Posterior middle frontal gyrus \\
\hline pMTG & Posterior middle temporal gyrus \\
\hline pSFG & Posterior superior frontal gyrus \\
\hline pSMG & Posterior supramarginal gyrus \\
\hline pSTG & Posterior superior temporal gyrus \\
\hline SPL & Superior parietal lobe \\
\hline trIFG & Triangular inferior frontal gyrus \\
\hline vPoG & Ventral postcentral gyrus \\
\hline vPrG & Ventral precentral gyrus \\
\hline
\end{tabular}

of $300 \mathrm{msec}$. Therefore, we additionally compared these 2 groups to each other with the objective of detecting the optimal PTI in combination with different ERTs.

\section{Comparison of $\mathrm{fMRI}$ and DCS}

After assigning the results of fMRI language mapping to the CPS, we compared them with the language sites defined by DCS as well, in the same manner in which we had compared the results of rTMS and DCS. Once more, we took the DCS data as the gold standard and compared the corresponding results of each patient and each region of the CPS. According to the comparison of rTMS and DCS, we documented the true positives and negatives, but without using an ERT. For example, if a CPS region was language positive during DCS mapping without a positive BOLD signal, we defined the region as a false negative in terms of fMRI for this patient; conversely, if a CPS region was language negative by DCS and language positive by fMRI, we counted this region as a false positive in terms of fMRI.

\section{Statistical Analysis}

For statistical analysis of the various comparisons, we summed up the obtained results of each patient, that is to say, the true and false positives and the true and false negatives and calculated the sensitivity, specificity, positive predictive value (PPV), and negative predictive value (NPV). This we did separately for each region of the CPS. ${ }^{51}$
To visualize and interpret the preserved data with the aim of figuring out an optimal protocol for the analysis of rTMS and for combined noninvasive language data, we issued ROC curves for all distinct kinds of analysis. In these ROC curves the sensitivity (y axis) is graphed against the formula $1-$ specificity (x axis). ${ }^{38}$

\section{Results}

Single Results of DCS, rTMS, and fMRI Language Mapping

Figure 1 outlines the total number of language-positive sites obtained by DCS (Fig. 1B) and rTMS (Fig. 1C), as well as the total number of stimulations implemented by the respective mapping technique in all patients. Accordingly, Fig. 1D shows the total number of positive BOLD signals per CPS region in all patients.

\section{Comparison of rTMS and DCS Language Mapping}

The mapping data of the 2 methods overlapped in 19 regions of the CPS. In total, we compared rTMS and DCS language mapping in 252 regions. Depending on the rTMS ERT, the true-positive results ranged from $28 \%$ (ERT 0\%) to $3 \%$ (ERT 50\%). As expected, the true-negative results increased from 10\% (ERT 0\%) to 68\% (ERT 50\%). Thus, the false-positive results decreased from $62 \%$ (ERT $0 \%$ ) to $3 \%$ (ERT 50\%). According to the decrease of true-positive results due to an enhanced ERT, the false-negative results increased from less than $1 \%$ (ERT 0\%) to 26\% (ERT 50\%). The overall ROC values for this comparison are illustrated in Table 3.

In addition, we compared the results of rTMS language mapping performed with a PTI of $0 \mathrm{msec}$ or $300 \mathrm{msec}$. In comparison with DCS, we achieved the highest sensitivity and the lowest rate of false positives by using an ERT of $15 \%, 20 \%, 25 \%$, and the 2-out-of- 3 rule in combination with a PTI of 0 msec (Fig. 2 and Table 4).

\section{Comparison of fMRI and DCS Language Mapping}

Across the 27 patients who were additionally examined by fMRI using an object-naming paradigm, the data overlapped with the intraoperative results in 22 regions of the CPS, and we compared these 2 methods in 258 regions altogether. In $11 \%$ of these comparisons we yielded a truepositive result and in $61 \%$ a true-negative result. Furthermore, we obtained false-positive results for $12 \%$ and falsenegative results for $16 \%$ of the comparisons. Subsequently, we calculated the ROCs for this comparison (Table 5). In comparison with rTMS language mapping with an ERT of $0 \%$ (Table 3), Table 5 shows the key advantages of fMRI language mapping reflected in its specificity.

\section{Additional Analysis Protocols for Combined Noninvasive Language Mapping \\ Protocol}

To combine the strengths of rTMS (high sensitivity and NPV) and fMRI language mapping (high specificity), we combined the results of both methods, using 2 distinct protocols for a combined noninvasive language mapping. The objective of the additional analysis Protocol 1 (A1) was to decrease the false-positive results of rTMS language map- 
TABLE 3. ROC for the comparison of rTMS ( 0 and $300 \mathrm{msec}$ PTI) and DCS language mapping*

\begin{tabular}{lcccc}
\hline \multicolumn{1}{c}{ rTMS ERT } & PPV & NPV & Sensitivity & Specificity \\
\hline 2 -out-of-3 rule & $34 \%$ & $79 \%$ & $67 \%$ & $49 \%$ \\
\hline$\geq 0 \%$ & $31 \%$ & $92 \%$ & $97 \%$ & $13 \%$ \\
\hline$\geq 5 \%$ & $31 \%$ & $84 \%$ & $92 \%$ & $18 \%$ \\
\hline$\geq 10 \%$ & $32 \%$ & $81 \%$ & $83 \%$ & $28 \%$ \\
\hline$\geq 15 \%$ & $30 \%$ & $74 \%$ & $63 \%$ & $43 \%$ \\
\hline$\geq 20 \%$ & $31 \%$ & $73 \%$ & $47 \%$ & $58 \%$ \\
\hline$\geq 25 \%$ & $33 \%$ & $74 \%$ & $40 \%$ & $67 \%$ \\
\hline$\geq 30 \%$ & $31 \%$ & $72 \%$ & $25 \%$ & $78 \%$ \\
\hline$\geq 35 \%$ & $35 \%$ & $73 \%$ & $18 \%$ & $87 \%$ \\
\hline$\geq 40 \%$ & $39 \%$ & $73 \%$ & $17 \%$ & $89 \%$ \\
\hline$\geq 45 \%$ & $45 \%$ & $73 \%$ & $13 \%$ & $94 \%$ \\
\hline$\geq 50 \%$ & $47 \%$ & $73 \%$ & $10 \%$ & $96 \%$ \\
\hline
\end{tabular}

* This table shows the sums of all mapped CPS regions for PPV, NPV, sensitivity, and specificity for the comparison of rTMS and DCS language mapping as functions of the rTMS ERT. The rTMS data for these calculations include both the 0 -msec and the 300 -msec PTI groups.

ping by qualifying them with fMRI-negative results. In contrast, the objective of the additional analysis Protocol 2 (A2) was to decrease fMRI's false-negative results by qualifying them with rTMS positive results. Table 6 gives a detailed overview of the adapted rules for both protocols. To evaluate the effect of the protocols, we compared the created assertions about language positivity and negativity A1 and A2 with the results of DCS language mapping.

\section{Results}

Equally to the comparison of rTMS and DCS language mapping and the comparison of fMRI and DCS language mapping, in this comparison, too, the DCS results were taken as gold standard for the calculation of the ROCs (Table 7). Moreover, we demonstrated the single results of each mapped CPS region regarding specificity and NPV for Protocol 1 (Fig. 3B and D) and sensitivity and PPV for Protocol 2 (Fig. 3A and C). According to the comparison of rTMS with DCS (Table 3), and to be more comparable with the comparison of fMRI with DCS (Table 5), the single results of Fig. 3 are analyzed with an ERT of 0\%.

Again, we observed the highest correlation to the results of DCS language mapping, regarding a high sensitivity and a low rate of false positives, for the combination of fMRI data and the results of rTMS language mappings performed with a PTI of $0 \mathrm{msec}$ and an ERT of $20 \%$ (Fig. 4).

\section{Discussion}

\section{rTMS Versus DCS Language Mapping}

Most importantly, it has to be stressed that the results of DCS are absolutely essential for language mapping in patients with left-sided perisylvian brain lesions and for brain mapping in its entirety. It should not be the aim to replace

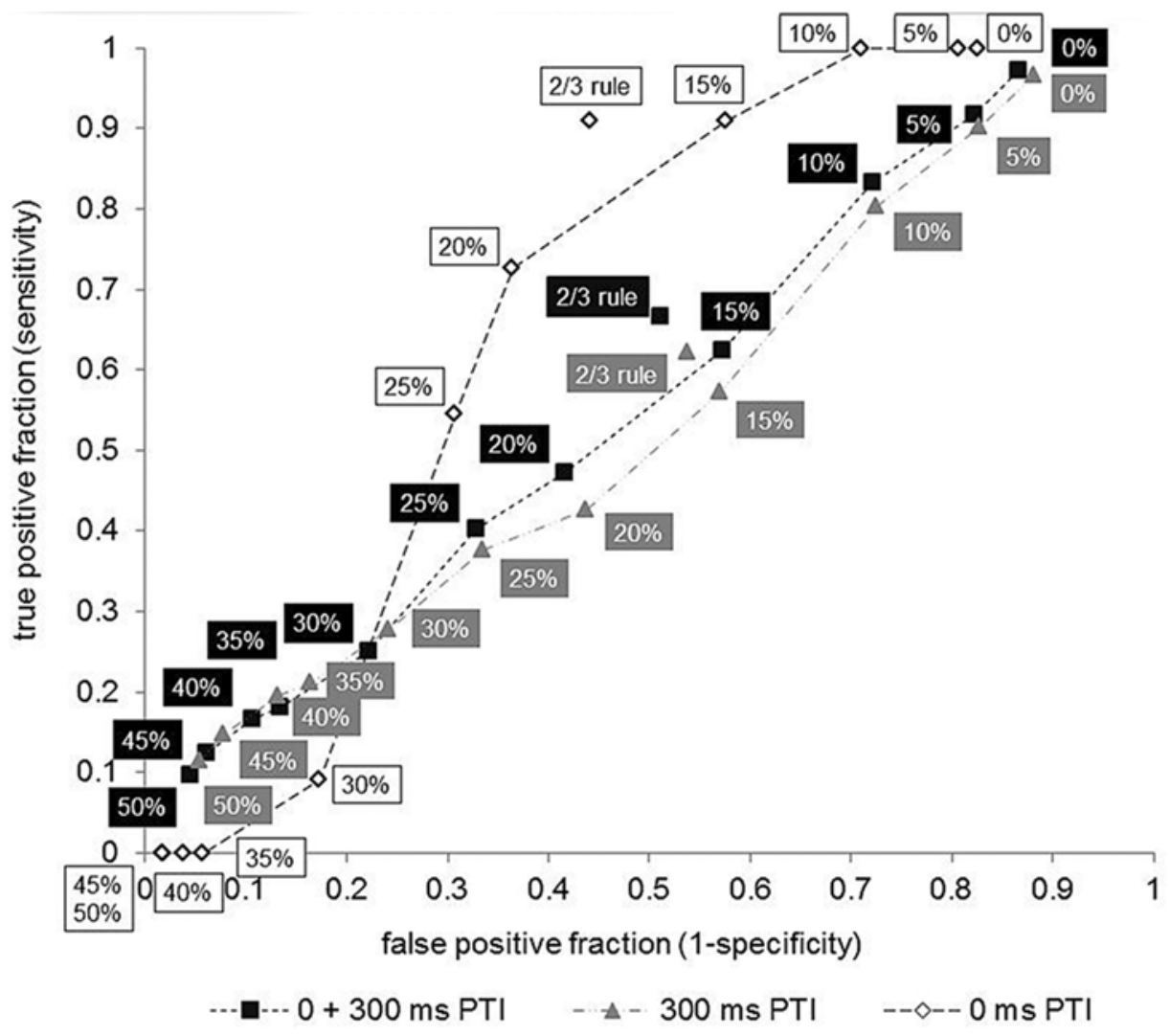

FIG. 2. ROC curves for the comparison of PTI. The results for the comparisons of rTMS language mapping performed with a PTI of 0 -msec and/or 300-msec with DCS language mapping are shown. The associated boxes describe the respective rTMS ERTs. 
TABLE 4. ROC for the comparison of rTMS (0 msec PTI) and DCS language mapping*

\begin{tabular}{lrrrc}
\hline \multicolumn{1}{c}{ rTMS ERT } & PPV & NPV & Sensitivity & Specificity \\
\hline 2-out-of-3 rule & $30 \%$ & $97 \%$ & $91 \%$ & $56 \%$ \\
\hline$\geq 0 \%$ & $20 \%$ & $100 \%$ & $100 \%$ & $17 \%$ \\
\hline$\geq 15 \%$ & $25 \%$ & $96 \%$ & $91 \%$ & $42 \%$ \\
\hline$\geq 20 \%$ & $30 \%$ & $92 \%$ & $73 \%$ & $63 \%$ \\
\hline$\geq 25 \%$ & $27 \%$ & $88 \%$ & $55 \%$ & $69 \%$ \\
\hline $250 \%$ & $0 \%$ & $82 \%$ & $0 \%$ & $98 \%$ \\
\hline
\end{tabular}

* This table highlights the results regarding the correlation of a high sensitivity and a low rate of false positives for the comparison between rTMS language mapping performed with a PTI of $0 \mathrm{msec}$ and DCS language mapping.

this gold standard, but to improve and advance the encouraging noninvasive technique rTMS with respect to preoperative assessment. For clinical usefulness, either the combination of a high specificity and NPV for the mapping of language-negative sites or the combination of a high sensitivity and PPV for the mapping of language-positive sites seems applicable. While negative mapping is sufficient for neurosurgical applications, positive mapping has to be aimed at for a general use in neuroscience..$^{30,51}$ The most basic difficulty of rTMS language mapping is determining language-positive sites accurately, at least in comparison with DCS. The constellation of a high sensitivity (overall [PTI 0 and $300 \mathrm{msec}$ ) 97\%, PTI 0 msec 100\%) but a low PPV (overall [PTI 0 and $300 \mathrm{msec}$ ] 31\%, PTI $0 \mathrm{msec}$ $20 \%$ ) using an ERT of $0 \%$ does not give sustainable information for neurosurgeons in the operating room and for basic researchers, respectively (Tables 3 and 4). In other words, rTMS language mapping is currently too sensitive for the mapping of language-positive sites. Most likely this is because it identifies not only language-eloquent but also language-involved cortical areas in general.

Like earlier studies, however, that have compared the results of preoperative rTMS language mapping with DCS during awake surgery, our study revealed a high sensitivity and NPV with an ERT of 0\% (Table 3). ${ }^{37,51,75}$ Most importantly, when regarding current protocols for DCS language mapping during awake surgery, some authors also rely only on negative language mapping intraoperatively. ${ }^{64,75} \mathrm{In}$ this respect, the absence of false-negative results, as revealed for rTMS language mapping performed with a PTI of 0 msec and analyzed with an ERT of $0 \%$, is of paramount importance (Table 4). ${ }^{15}$ Thus, the high sensitivity and NPV of our presented protocol seem already applicable in the daily clinical routine. ${ }^{37}$ These values promise very reliable negative results and accordingly enable a more extensive resection, which has proved crucial in neurooncology.6,71 In addition, our analysis of different ERTs shows that

TABLE 5. ROC for the comparison of $\mathrm{FMRI}$ and DCS

\begin{tabular}{cc}
\hline Variable & Value \\
\hline PPV & $48 \%$ \\
\hline NPV & $79 \%$ \\
\hline Sensitivity & $40 \%$ \\
\hline Specificity & $84 \%$ \\
\hline
\end{tabular}

TABLE 6. Definitions of the protocols for additional analysis*

\begin{tabular}{cccc}
\hline Protocol & Result of rTMS & Result of fMRI & $\begin{array}{c}\text { Definition in Additional } \\
\text { Analysis Protocol }\end{array}$ \\
\hline 1 (A1) & + & + & A1-positive \\
\cline { 2 - 4 } & - & - & A1-negative \\
\cline { 2 - 4 } & + & - & A1-negative \\
\hline 2 (A2) & - & + & A1-negative \\
& + & + & A2-positive \\
\cline { 2 - 4 } & + & - & A2-negative \\
\hline + = language positive; - = language negative. & A2-positive \\
* This table gives an overview of the rules for the 2 protocols with the intent of \\
a combined noninvasive language-mapping protocol. The objective of Protocol \\
1 was to decrease rTMS false-positive results by qualifying them with fMRI \\
negative results. The objective of Protocol 2 was to decrease fMRI false- \\
negative results by qualifying them with rTMS positive results.
\end{tabular}

rTMS provides sufficient negative language maps (specificity: overall [PTI 0 and $300 \mathrm{msec}$ ] 96\%; PTI 0 msec 98\% and NPV overall [PTI 0 and $300 \mathrm{msec}$ ] 73\%; PTI $0 \mathrm{msec}$ $82 \%$ ) compared with DCS language mapping, by analyzing the results with a high ERT $(\geq 50 \%)$ (Fig. 2 and Tables 3 and 4).

Even particularly with regard to negative language mapping, because of the increase of true-negative results, we could show a reduction in false-positive results by performing rTMS language mapping with a PTI of 0 msec according to a recent report. ${ }^{30}$ We therefore compared rTMS language mapping with PTIs of $0 \mathrm{msec}$ and $300 \mathrm{msec}$. The obviously higher correlation of the immediate rTMS stimulation beginning at the same time as the picture presentation could confirm other noninvasive data (Fig. 2 and Tables 3 and 4). ${ }^{30}$ This analysis showed the highest correlations for sensitivity, combined with a low rate of falsepositive results and a high NPV, using ERTs of 15\% (sensitivity $91 \%$, NPV 96\%), $20 \%$ (sensitivity $73 \%$, NPV 92\%), $25 \%$ (sensitivity 55\%, NPV $88 \%$ ), and the 2 -out-of-3 rule (sensitivity 91\%, NPV 97\%) (Fig. 2 and Table 4). These ERTs should be the basis for further research to refine the promising method of rTMS in language mapping.

Nevertheless, for the comparison of rTMS and DCS language mapping, the occurrence of false-positive results has to be discussed. But are these false positives truly false positives? Yes, they are, regarding the wealth of experience of DCS and its status as the gold standard for the detection of language-eloquent brain regions. ${ }^{7,9,26,44,45,62,65}$ Noninvasive mapping techniques for identifying human language function have to compete with results mapped during awake surgery. Having no other modalities, however, we still do not know exactly the role played by these DCS-identified language-positive points. Since resection of these areas is associated mostly with a consecutive and at least transient language deficit, we have to name them language eloquent. $16,23,41,54$ Since rTMS is very sensitive, language-positive cortical areas as identified by rTMS are most likely not only language eloquent but are also involved in language function in general. This can also be taken into account regarding the false-positive results of 
TABLE 7. Results for the combined fMRI/rTMS language mapping according to Protocol 1 and Protocol 2 compared with DCS language mapping*

\begin{tabular}{|c|c|c|c|c|c|c|c|c|}
\hline \multirow[b]{2}{*}{ rTMS ERT } & \multicolumn{4}{|c|}{ Protocol 1} & \multicolumn{4}{|c|}{ Protocol 2} \\
\hline & PPV & NPV & Sensitivity & Specificity & PPV & NPV & Sensitivity & Specificity \\
\hline 2-out-of-3 rule & $55 \%$ & $75 \%$ & $33 \%$ & $88 \%$ & $35 \%$ & $77 \%$ & $73 \%$ & $40 \%$ \\
\hline$\geq 0 \%$ & $51 \%$ & $76 \%$ & $41 \%$ & $83 \%$ & $34 \%$ & $95 \%$ & $98 \%$ & $13 \%$ \\
\hline$\geq 5 \%$ & $50 \%$ & $75 \%$ & $38 \%$ & $83 \%$ & $34 \%$ & $89 \%$ & $95 \%$ & $17 \%$ \\
\hline$\geq 10 \%$ & $52 \%$ & $75 \%$ & $36 \%$ & $85 \%$ & $34 \%$ & $82 \%$ & $88 \%$ & $25 \%$ \\
\hline$\geq 15 \%$ & $54 \%$ & $74 \%$ & $30 \%$ & $89 \%$ & $34 \%$ & $75 \%$ & $72 \%$ & $37 \%$ \\
\hline$\geq 20 \%$ & $52 \%$ & $72 \%$ & $20 \%$ & $92 \%$ & $36 \%$ & $76 \%$ & $66 \%$ & $48 \%$ \\
\hline$\geq 25 \%$ & $63 \%$ & $72 \%$ & $19 \%$ & $95 \%$ & $38 \%$ & $76 \%$ & $61 \%$ & $55 \%$ \\
\hline$\geq 30 \%$ & $70 \%$ & $71 \%$ & $11 \%$ & $98 \%$ & $40 \%$ & $76 \%$ & $55 \%$ & $63 \%$ \\
\hline$\geq 35 \%$ & $75 \%$ & $71 \%$ & $9 \%$ & $99 \%$ & $43 \%$ & $76 \%$ & $50 \%$ & $70 \%$ \\
\hline$\geq 40 \%$ & $71 \%$ & $71 \%$ & $8 \%$ & $99 \%$ & $44 \%$ & $76 \%$ & $50 \%$ & $72 \%$ \\
\hline$\geq 45 \%$ & $67 \%$ & $70 \%$ & $6 \%$ & $99 \%$ & $48 \%$ & $77 \%$ & $48 \%$ & $76 \%$ \\
\hline$\geq 50 \%$ & $67 \%$ & $70 \%$ & $3 \%$ & $99 \%$ & $48 \%$ & $77 \%$ & $48 \%$ & $77 \%$ \\
\hline
\end{tabular}

* The data include the results of all mapped CPS regions and both PTI groups.

rTMS language mapping. The high sensitivity concerning language-involved regions might also be reflected in the evocation of language errors in CPS regions defined as language negative by DCS. The rTMS ERs of these regions are largely on an intermediate level, while a relatively small number of stimulations were delivered to them by DCS. Additionally, the distribution of CPS regions with a high ER is similar on both the rTMS and DCS language maps (Fig. 1B and C). Yet, this explanation of false-posi- tive results and high ERs can only be contemplated for the total language maps, since the overall rate of false positives is calculated by the sums of results of all patients. Nevertheless, both techniques operate on the same theory-the "virtual lesion"-and rTMS has already proved effective concerning language tasks. ${ }^{14,19,78,81}$ In other words, rTMS language mapping is already feasible, but there are some differences regarding the comparison with DCS language mapping.

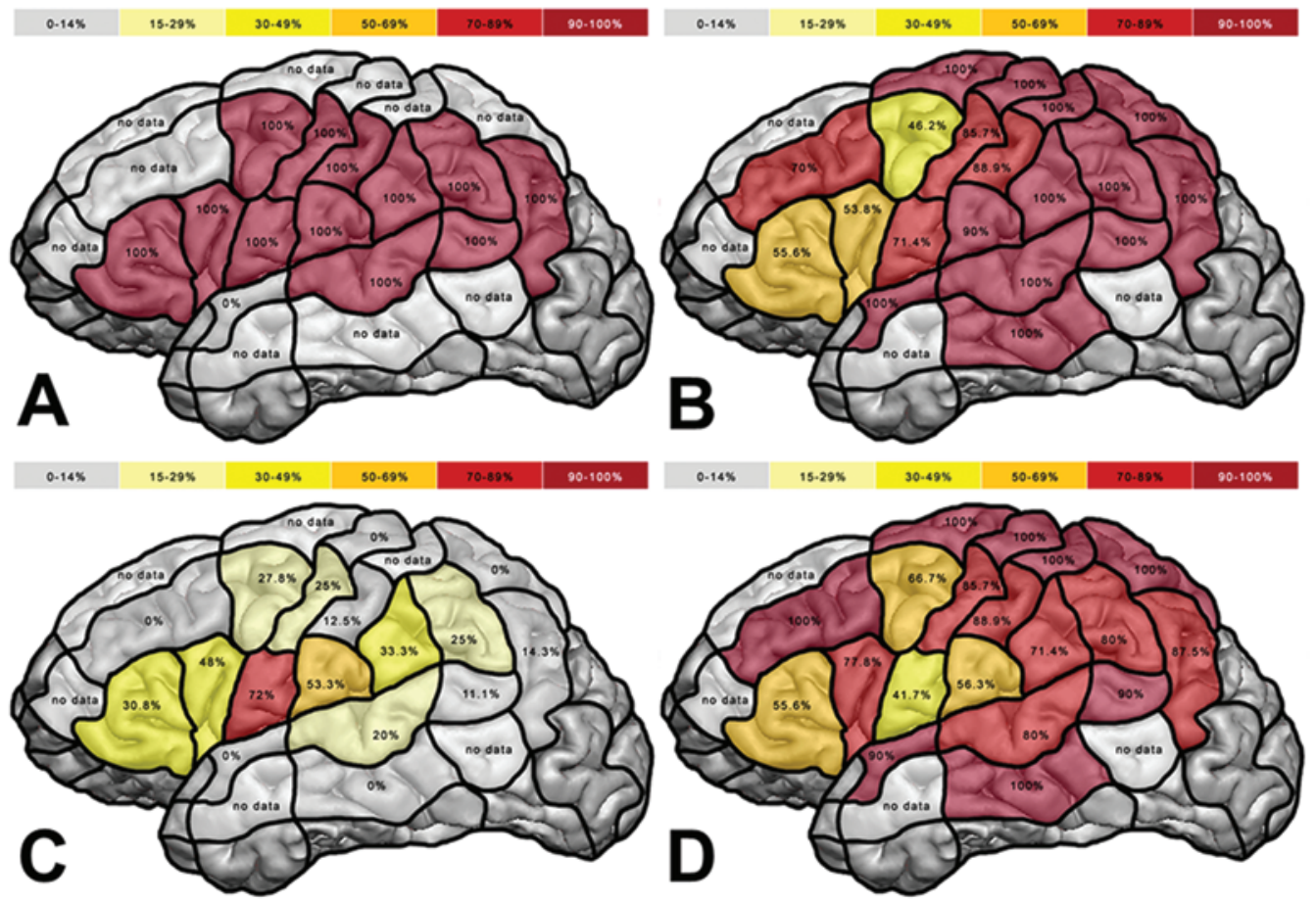

FIG. 3. Combined fMRI/rTMS (ERT 0\%, 0-msec and 300-msec PTI) language mapping compared with DCS. The schemes show the results for each mapped CPS regions for the comparison of combined fMRI/rTMS language mapping against DCS language mapping. For Protocol 1 we demonstrate specificity (B) and NPV (D). The schemes for Protocol 2 show sensitivity (A) and PPV (C). The TMS part of both protocols has been analyzed with an ERT of $0 \%$, and the results include both PTI groups $(0$-msec and 300-msec PTI). 


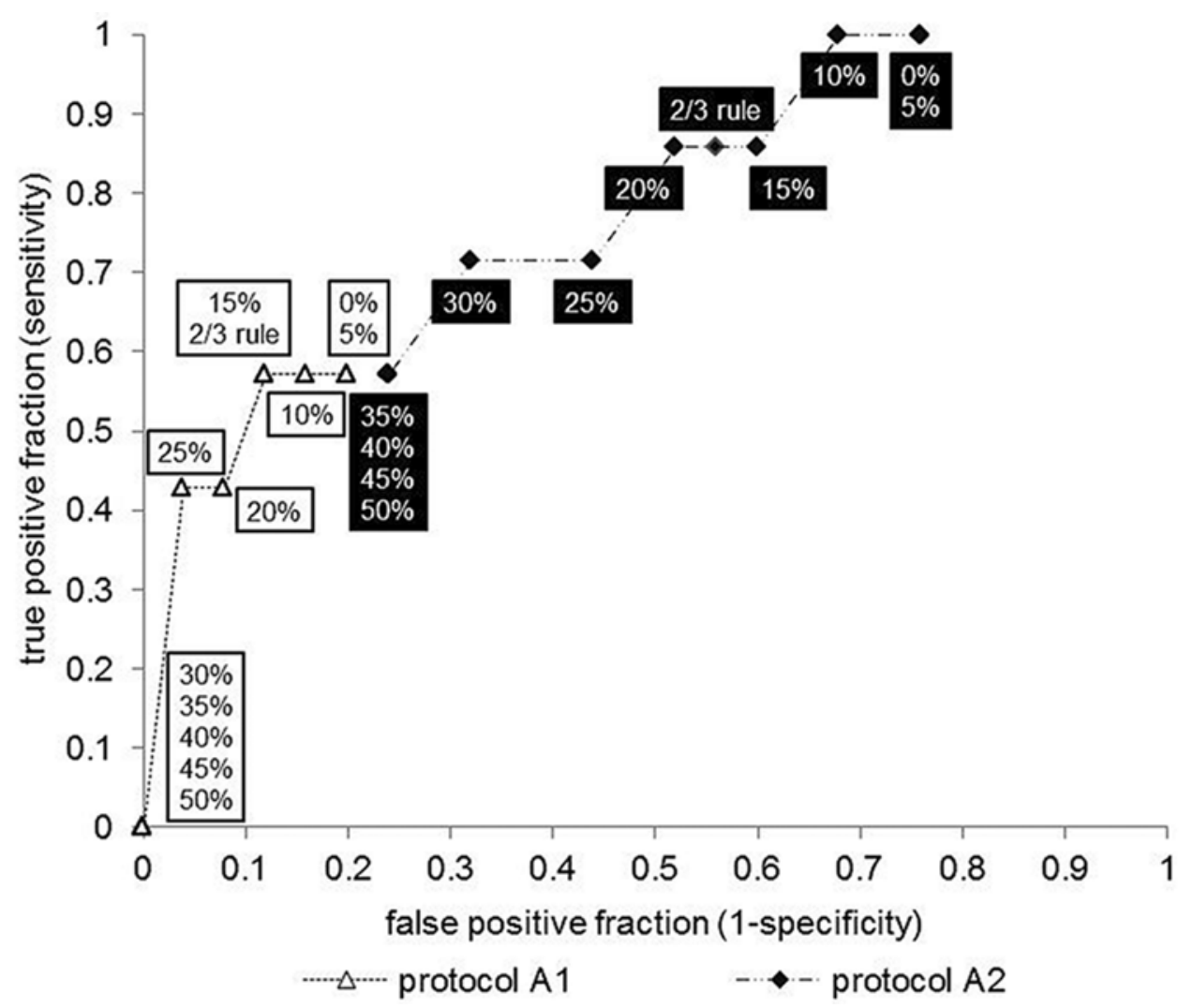

FIG. 4. ROC curves for combined noninvasive language mapping. ROC curves for the comparison of Protocols 1 and 2 with DCS language mapping are shown. The rTMS language mappings leading to these results were performed with a PTI of 0 msec. The associated boxes describe the rTMS ERTs.

Furthermore, the classic distribution of human language function to Broca's and Wernicke's areas is no longer current, and it is even not definitely resolved how essential these 2 regions are. ${ }^{2,5,44}$ Several studies assume and partially prove that human language function is organized in a complex network with the possibility of reorganization. ${ }^{10,16,30,31,46,54}$ In this context it may be appropriate to ask whether rTMS maps language function more precisely than DCS. It is definitely easier to analyze language errors using the video data recorded during rTMS language mapping than to detect language errors in the operating room, although the neuropsychologist can concentrate exclusively on evaluating language performance during awake surgery. For the analysis of rTMS language mapping, unclear sequences can be reviewed several times; in comparison with baseline, moreover, the pronunciation is more distinct, and the setting is less stressful for the patient. This may be a reason for the occurrence of errors in rTMS language maps, which perhaps do not define essential language sites, but rather define regions participating in language-related networks that are involved in language production but may not be essential. Thus, applying rTMS to these stimulations may cause only minor but detectable language impairment. That the resection of some of these sites does not lead inevitably to a permanent postoperative deficit is comparable to the results of former studies concerning the resection of positive language sites defined by DCS. $16,23,41,51,54$
Another difference may be the impact of rTMS on functional connectivity, since rTMS may affect subcortical pathways more significantly than bipolar DCS. . $^{17,25,41,64,73}$ This may be a reason for rTMS producing more false-positive results than DCS. On the other hand, the subcortical affection by rTMS may be an approach toward the mapping of language-positive sites in the future. To enable a safer resection by preoperative mapping even of deeper-located parts, the subcortical affection should be verified by the results of diffusion tensor imaging fiber tracking, even if this technique also has to be further investigated..$^{15,39}$

\section{Combined fMRI and rTMS Language Mapping}

Despite the already encouraging results of rTMS language mapping in comparison with DCS, especially with a PTI of $0 \mathrm{msec}$, we combined the results of rTMS and fMRI language mapping. This was done to combine the advantages of each method based on the 2 methods' respective comparisons with DCS language mapping. In addition to the aforementioned results of rTMS, we revealed a high specificity (84\%) for the comparison of fMRI and DCS, as other studies have done before ${ }^{57}$ (Tables 3 and 5).

The objective of Protocol 1 was to decrease rTMS's false-positive results (Table 6). On the one hand, specificity and PPV greatly increased, since many of the initially false-positive results could be unmasked as true negatives (Fig. 3B and Table 7). But there was also a huge decrease in sensitivity and NPV in comparison with the sole use 
of rTMS (Table 3 vs Table 7; Fig. 3D). This result suggests that fMRI's negative spots additionally masked some of rTMS's true-positive results. Nevertheless, as Fig. 3B shows, Protocol 1 provides sufficient negative language maps in comparison with DCS.

Accordingly, the effect of decreasing fMRI false-negative results by using Protocol 2 is impressive regarding sensitivity and NPV compared with the sole use of fMRI language mapping (Fig. 3A; Table 5 vs Table 7). But as Protocol 1 transferred some of the initially true-positive rTMS results into false negatives (Fig. 3D; Table 3 vs Table 7), Protocol 2 transferred some of the fMRI's true-negative results into false positives (Fig. 3C; Table 5 vs Table 7). Even Protocol 2, however, yields sufficient language maps to map negative sites (Table 7).

At first view, the use of Protocol 1 or 2 does not bring advantages in comparison with language mapping using rTMS alone. The mapping of language-negative sites renders comparable results for specificity and NPV for Protocol 1 (Fig. 3B and D and Table 7) and the sole use of rTMS language mapping with an ERT of 50\% (Table 3). Yet the PPVs of Protocol 1 are constantly equal to or greater than $50 \%$, and even up to $75 \%$ (Table 7). More importantly, the specificities of Protocol 1 are greater than $80 \%$ across all ERTs. Thus, Protocol 2 shows comparable results for sensitivity and NPV as well as rTMS language mapping alone (Table 3 vs 7; Fig. 3A). But the sensitivities do not decrease similarly, especially for higher ERTs.

Although several studies have found an incomplete match between fMRI and DCS language mapping, fMRI is still the most distributed modality for noninvasive language mapping..$^{21,24,57,68,82}$ This status similarly testifies to an extensive experience, not least in the field of language mapping. Yet a review of studies about the mapping of language function by fMRI has concluded that even this well-established technique has to be further refined; another study has confirmed the selection of a more aggressive therapeutic approach. ${ }^{4,49}$ Still, as other studies have concluded, the results of fMRI and DCS will never completely agree, because the 2 methods have fundamental differences. ${ }^{60,68}$

As already mentioned, the quest of mapping human language function lies in its complexity and associated networks. With our protocols for combined noninvasive language mapping, we pay attention to this issue. The strength of fMRI is the visualization of cortical networks, whereas the mapping of language function by rTMS seems to be more targeted at a higher spatial resolution, as has been shown for cortical motor function. ${ }^{22,34}$ The basic principle of fMRI is the assumption that task-related brain function and its related neural activity can be measured by the effect of increases in deoxyhemoglobin from activated neurons consuming more oxygen. These BOLD signals therefore visualize activated cortical regions. ${ }^{43}$ It has to be mentioned, however, that the destruction of a formerly activated region does not automatically cause a deficit in language function. ${ }^{17,24}$ In contrast, rTMS mapping shows the effect of a temporary functional lesion in the depolarization and therefore inactivation of a designated brain region and the whole connected functional network. 14,19,47,79,81 A recently reported case showed fMRI and rTMS yielding contrary results, but the use of our protocols would make it possible to combine lesion-based and blood flow-based techniques even in this case. ${ }^{68}$ That these 2 principles can complement each other has already been shown in previous studies. ${ }^{61,81}$ Moreover, as other authors, surgeons in particular, suggest and already perform consecutive awake surgery in oncological patients, combined language mapping by fMRI and rTMS makes it possible to back this approach up and to support the oncological considerations by longitudinal noninvasive follow-up examinations for the inclusion of plastic reshaping of cortical language function. $11,16,17,20,42,54,76$ Of course, the 2 protocols, being mutually exclusive, cannot be applied at the same time. Nevertheless, the results show that the combination of the results of fMRI and rTMS language mapping is able to achieve a high sensitivity and a high specificity.

In summary, with our present data we contribute to the development of more standardized protocols for both the performance and the analysis of rTMS language mapping. In addition, we have evaluated 2 new protocols for the combined application of rTMS and fMRI, to ensure a safer and more reliable preoperative language mapping.

\section{Limitations}

One of the general limitations of preoperative mapping is the effect of brain shift after the durotomy. ${ }^{27,72}$ This may be a reason for slightly differing results from mapping that was done before surgery and during surgery. Yet the intraoperative location of DCS-positive points has also been detected by pial venous structures allowing the identification of the correct CPS region after durotomy. ${ }^{35}$ The CPS per se might be a further limitation of our study, since the error margins are larger than $10 \mathrm{~mm}$, while it can be assumed that the spatial resolution of DCS is even smaller than 10 $\mathrm{mm} .{ }^{23}$ In this study we used the CPS for statistical analysis to combine the 2 noninvasive techniques with the gold standard. Still, our present results should be reproduced in any case, and using optimized systems for the comparisons should be considered in the future. Another limitation of our study is the sole use of an object-naming task for both preoperative mapping techniques. This limitation should not affect the comparisons with DCS language mapping, since we have also used an object-naming task for the intraoperative language mapping. Yet, in the basic research of language function and the further refining of our protocols, it is especially tasks involving comprehension of language that should be applied. The object-naming task, however, is able to reproduce the whole process of word production and incorporates all presumably language-eloquent brain regions. ${ }^{13,17,30,66}$ This has been shown in several studies and for each of the 3 modalities. ${ }^{9,30,40}$

However, it has also been shown that fMRI language mapping is more sensitive to anterior than to posterior language-related cortical regions. ${ }^{3}$ The more frequently detected occurrence of positive BOLD signals within the anterior language-related regions could be approved by our study and these circumstances have to be considered in case of assessing the accuracy of fMRI language mapping (Fig. 1D).

What is more, examination by fMRI depends heavily on the patient's compliance. ${ }^{1,41,47,57,70}$ Of course, compliance always plays an important role in the analysis of hu- 
man brain function, especially regarding the detection of language-eloquent regions by DCS. ${ }^{73}$ But the examiners cannot control compliance during the object-naming task in the MR scanner. In contrast, a lack of cooperation is immediately noticeable during the rTMS language mapping. This has to be considered when combining the results of rTMS and fMRI language mapping.

On the other hand and in the consideration of the 2 noninvasive methods, it has to be mentioned that rTMS language mapping is currently still more time-consuming, costlier, and less standardized than fMRI. However, especially in the present study, we refine this promising and aspiring technique, among others, and provide data toward a higher grade of standardization to face these shortcomings.

In addition, the 2 PTI groups (0-msec and 300-msec PTI) have not been randomized. Even though a recently published study has proved the advantages of rTMS language mapping performed with a PTI of 0 msec, and our results regarding this analysis are like theirs, this fact must still be noted as a limitation of our study. ${ }^{30}$

\section{Conclusions}

The results of our study show that the raw data of rTMS language mapping should be analyzed with an ERT of $15 \%, 20 \%, 25 \%$, or the 2 -out-of-3 rule to obtain a high prediction of DCS language mapping. Moreover, we can support previous data suggesting that rTMS language mapping should be performed with a PTI of 0 msec. We can also conclude that combining the more sensitive technique of rTMS and the more specific technique of fMRI leads to a higher correlation to DCS language mapping than either technique alone. With this in mind, our presented Protocols 1 and 2 provide a promising noninvasive approach that could be helpful for language-mapping assessment prior to the gold standard of intraoperative DCS.

\section{References}

1. Abou-Khalil B: An update on determination of language dominance in screening for epilepsy surgery: the Wada test and newer noninvasive alternatives. Epilepsia 48:442-455, 2007

2. Baum SH, Martin RC, Hamilton AC, Beauchamp MS: Multisensory speech perception without the left superior temporal sulcus. Neuroimage 62:1825-1832, 2012

3. Bizzi A, Blasi V, Falini A, Ferroli P, Cadioli M, Danesi U, et al: Presurgical functional MR imaging of language and motor functions: validation with intraoperative electrocortical mapping. Radiology 248:579-589, 2008

4. Bookheimer S: Pre-surgical language mapping with functional magnetic resonance imaging. Neuropsychol Rev 17:145-155, 2007

5. Briganti C, Sestieri C, Mattei PA, Esposito R, Galzio RJ, Tartaro A, et al: Reorganization of functional connectivity of the language network in patients with brain gliomas. AJNR Am J Neuroradiol 33:1983-1990, 2012

6. Capelle L, Fontaine D, Mandonnet E, Taillandier L, Golmard JL, Bauchet L, et al: Spontaneous and therapeutic prognostic factors in adult hemispheric World Health Organization Grade II gliomas: a series of 1097 cases. Clinical article. J Neurosurg 118:1157-1168, 2013

7. Chan-Seng E, Moritz-Gasser S, Duffau H: Awake mapping for low-grade gliomas involving the left sagittal stratum: anatomofunctional and surgical considerations. J Neurosurg 120:1069-1077, 2014

8. Corina DP, Gibson EK, Martin R, Poliakov A, Brinkley J, Ojemann GA: Dissociation of action and object naming: evidence from cortical stimulation mapping. Hum Brain Mapp 24:1-10, 2005

9. Corina DP, Loudermilk BC, Detwiler L, Martin RF, Brinkley JF, Ojemann G: Analysis of naming errors during cortical stimulation mapping: implications for models of language representation. Brain Lang 115:101-112, 2010

10. Davidson DJ, Indefrey P: Error-related activity and correlates of grammatical plasticity. Front Psychol 2:219, 2011

11. De Benedictis A, Moritz-Gasser S, Duffau H: Awake mapping optimizes the extent of resection for low-grade gliomas in eloquent areas. Neurosurgery 66:1074-1084, 2010

12. De Witt Hamer PC, Hendriks EJ, Mandonnet E, Barkhof F, Zwinderman AH, Duffau H: Resection probability maps for quality assessment of glioma surgery without brain location bias. PLoS ONE 8:e73353, 2013

13. DeLeon J, Gottesman RF, Kleinman JT, Newhart M, Davis $\mathrm{C}$, Heidler-Gary J, et al: Neural regions essential for distinct cognitive processes underlying picture naming. Brain 130:1408-1422, 2007

14. Devlin JT, Watkins KE: Stimulating language: insights from TMS. Brain 130:610-622, 2007

15. Duffau H: Diffusion Tensor imaging is a research and educational tool, but not yet a clinical tool. World Neurosurg 82:e43-e45, 2014

16. Duffau H: New concepts in surgery of WHO grade II gliomas: functional brain mapping, connectionism and plasticity-a review. J Neurooncol 79:77-115, 2006

17. Duffau H, Moritz-Gasser S, Mandonnet E: A re-examination of neural basis of language processing: proposal of a dynamic hodotopical model from data provided by brain stimulation mapping during picture naming. Brain Lang 131:1-10, 2014

18. Epstein CM: Transcranial magnetic stimulation: language function. J Clin Neurophysiol 15:325-332, 1998

19. Epstein CM, Lah JJ, Meador K, Weissman JD, Gaitan LE, Dihenia B: Optimum stimulus parameters for lateralized suppression of speech with magnetic brain stimulation. Neurology 47:1590-1593, 1996

20. Findlay AM, Ambrose JB, Cahn-Weiner DA, Houde JF, Honma S, Hinkley LB, et al: Dynamics of hemispheric dominance for language assessed by magnetoencephalographic imaging. Ann Neurol 71:668-686, 2012

21. FitzGerald DB, Cosgrove GR, Ronner S, Jiang H, Buchbinder BR, Belliveau JW, et al: Location of language in the cortex: a comparison between functional MR imaging and electrocortical stimulation. AJNR Am J Neuroradiol 18:1529-1539, 1997

22. Forster MT, Hattingen E, Senft C, Gasser T, Seifert V, Szelényi A: Navigated transcranial magnetic stimulation and functional magnetic resonance imaging: advanced adjuncts in preoperative planning for central region tumors. Neurosurgery 68:1317-1325, 2011

23. Gil-Robles S, Duffau H: Surgical management of World Health Organization Grade II gliomas in eloquent areas: the necessity of preserving a margin around functional structures. Neurosurg Focus 28(2):E8, 2010

24. Giussani C, Roux FE, Ojemann J, Sganzerla EP, Pirillo D, Papagno C: Is preoperative functional magnetic resonance imaging reliable for language areas mapping in brain tumor surgery? Review of language functional magnetic resonance imaging and direct cortical stimulation correlation studies. Neurosurgery 66:113-120, 2010

25. Guggisberg AG, Honma SM, Findlay AM, Dalal SS, Kirsch HE, Berger MS, et al: Mapping functional connectivity in patients with brain lesions. Ann Neurol 63:193-203, 2008 
26. Haglund MM, Berger MS, Shamseldin M, Lettich E, Ojemann GA: Cortical localization of temporal lobe language sites in patients with gliomas. Neurosurgery 34:567-576, 1994

27. Hastreiter P, Rezk-Salama C, Soza G, Bauer M, Greiner G, Fahlbusch R, et al: Strategies for brain shift evaluation. Med Image Anal 8:447-464, 2004

28. Huber W, Weniger D, Poeck K, Willmes K: [The Aachen Aphasia Test Rationale and construct validity (author's transl).]. Nervenarzt 51:475-482, 1980 (Ger)

29. Ilmoniemi RJ, Ruohonen J, Karhu J: Transcranial magnetic stimulation-a new tool for functional imaging of the brain. Crit Rev Biomed Eng 27:241-284, 1999

30. Indefrey P: The spatial and temporal signatures of word production components: a critical update. Front Psychol 2:255, 2011

31. Ius T, Angelini E, Thiebaut de Schotten M, Mandonnet E, Duffau H: Evidence for potentials and limitations of brain plasticity using an atlas of functional resectability of WHO grade II gliomas: towards a "minimal common brain." Neuroimage 56:992-1000, 2011

32. Kohn SE, Goodglass H: Picture-naming in aphasia. Brain Lang 24:266-283, 1985

33. Krieg SM, Sabih J, Bulubasova L, Obermueller T, Negwer $\mathrm{C}$, Janssen I, et al: Preoperative motor mapping by navigated transcranial magnetic brain stimulation improves outcome for motor eloquent lesions. Neuro Oncol 16:1274-1282, 2014

34. Krieg SM, Shiban E, Buchmann N, Gempt J, Foerschler A, Meyer B, et al: Utility of presurgical navigated transcranial magnetic brain stimulation for the resection of tumors in eloquent motor areas. J Neurosurg 116:994-1001, 2012

35. Krieg SM, Shiban E, Buchmann N, Meyer B, Ringel F: Presurgical navigated transcranial magnetic brain stimulation for recurrent gliomas in motor eloquent areas. Clin Neurophysiol 124:522-527, 2013

36. Krieg SM, Sollmann N, Hauck T, Ille S, Foerschler A, Meyer $\mathrm{B}$, et al: Functional language shift to the right hemisphere in patients with language-eloquent brain tumors. PLoS ONE 8:e75403, 2013

37. Krieg SM, Sollmann N, Hauck T, Ille S, Meyer B, Ringel F: Repeated mapping of cortical language sites by preoperative navigated transcranial magnetic stimulation compared to repeated intraoperative DCS mapping in awake craniotomy. BMC Neurosci 15:20, 2014

38. Lalkhen AG, McCluskey A: Clinical tests: sensitivity and specificity. Contin Educ Anaesth Crit Care Pain 8:221223, 2008

39. Leclercq D, Duffau H, Delmaire C, Capelle L, Gatignol P, Ducros M, et al: Comparison of diffusion tensor imaging tractography of language tracts and intraoperative subcortical stimulations. J Neurosurg 112:503-511, 2010

40. Lioumis P, Zhdanov A, Mäkelä N, Lehtinen H, Wilenius J, Neuvonen T, et al: A novel approach for documenting naming errors induced by navigated transcranial magnetic stimulation. J Neurosci Methods 204:349-354, 2012

41. Martino J, Honma SM, Findlay AM, Guggisberg AG, Owen JP, Kirsch HE, et al: Resting functional connectivity in patients with brain tumors in eloquent areas. Ann Neurol 69:521-532, 2011

42. Martino J, Taillandier L, Moritz-Gasser S, Gatignol P, Duffau $\mathrm{H}$ : Re-operation is a safe and effective therapeutic strategy in recurrent WHO grade II gliomas within eloquent areas. Acta Neurochir (Wien) 151:427-436, 2009

43. Ogawa S, Tank DW, Menon R, Ellermann JM, Kim SG, Merkle $\mathrm{H}$, et al: Intrinsic signal changes accompanying sensory stimulation: functional brain mapping with magnetic resonance imaging. Proc Natl Acad Sci U S A 89:59515955,1992

44. Ojemann G, Ojemann J, Lettich E, Berger M: Cortical lan- guage localization in left, dominant hemisphere. An electrical stimulation mapping investigation in 117 patients. J Neurosurg 71:316-326, 1989

45. Ojemann GA, Whitaker HA: Language localization and variability. Brain Lang 6:239-260, 1978

46. Papagno C, Gallucci M, Casarotti A, Castellano A, Falini A, Fava E, et al: Connectivity constraints on cortical reorganization of neural circuits involved in object naming. Neuroimage 55:1306-1313, 2011

47. Pascual-Leone A, Bartres-Faz D, Keenan JP: Transcranial magnetic stimulation: studying the brain-behaviour relationship by induction of "virtual lesions.. Philos Trans R Soc Lond B Biol Sci 354:1229-1238, 1999

48. Pascual-Leone A, Gates JR, Dhuna A: Induction of speech arrest and counting errors with rapid-rate transcranial magnetic stimulation. Neurology 41:697-702, 1991

49. Petrella JR, Shah LM, Harris KM, Friedman AH, George TM, Sampson JH, et al: Preoperative functional MR imaging localization of language and motor areas: effect on therapeutic decision making in patients with potentially resectable brain tumors. Radiology 240:793-802, 2006

50. Picht T, Kombos T, Gramm HJ, Brock M, Suess O: Multimodal protocol for awake craniotomy in language cortex tumour surgery. Acta Neurochir (Wien) 148:127-138, 2006

51. Picht T, Krieg SM, Sollmann N, Rösler J, Niraula B, Neuvonen T, et al: A comparison of language mapping by preoperative navigated transcranial magnetic stimulation and direct cortical stimulation during awake surgery. Neurosurgery 72:808-819, 2013

52. Picht T, Mularski S, Kuehn B, Vajkoczy P, Kombos T, Suess $\mathrm{O}$ : Navigated transcranial magnetic stimulation for preoperative functional diagnostics in brain tumor surgery. Neurosurgery 65 (6 Suppl):93-99, 2009

53. Picht T, Schmidt S, Brandt S, Frey D, Hannula H, Neuvonen $\mathrm{T}$, et al: Preoperative functional mapping for rolandic brain tumor surgery: comparison of navigated transcranial magnetic stimulation to direct cortical stimulation. Neurosurgery 69:581-588, 2011

54. Robles SG, Gatignol P, Lehéricy S, Duffau H: Long-term brain plasticity allowing a multistage surgical approach to World Health Organization Grade II gliomas in eloquent areas. J Neurosurg 109:615-624, 2008

55. Rogić M, Deletis V, Fernández-Conejero I: Inducing transient language disruptions by mapping of Broca's area with modified patterned repetitive transcranial magnetic stimulation protocol. J Neurosurg 120:1033-1041, 2014

56. Rossi S, Hallett M, Rossini PM, Pascual-Leone A: Safety, ethical considerations, and application guidelines for the use of transcranial magnetic stimulation in clinical practice and research. Clin Neurophysiol 120:2008-2039, 2009

57. Roux FE, Boulanouar K, Lotterie JA, Mejdoubi M, LeSage JP, Berry I: Language functional magnetic resonance imaging in preoperative assessment of language areas: correlation with direct cortical stimulation. Neurosurgery 52:13351347,2003

58. Ruohonen J, Ilmoniemi RJ: Modeling of the stimulating field generation in TMS. Electroencephalogr Clin Neurophysiol Suppl 51:30-40, 1999

59. Ruohonen J, Karhu J: Navigated transcranial magnetic stimulation. Neurophysiol Clin 40:7-17, 2010

60. Rutten GJ, Ramsey NF: The role of functional magnetic resonance imaging in brain surgery. Neurosurg Focus 28(2):E4, 2010

61. Sack AT, Cohen Kadosh R, Schuhmann T, Moerel M, Walsh $\mathrm{V}$, Goebel R: Optimizing functional accuracy of TMS in cognitive studies: a comparison of methods. J Cogn Neurosci 21:207-221, 2009

62. Sacko O, Lauwers-Cances V, Brauge D, Sesay M, Brenner A, Roux FE: Awake craniotomy vs surgery under general anes- 
thesia for resection of supratentorial lesions. Neurosurgery 68:1192-1199, 2011

63. Salmelin R, Helenius P, Service E: Neurophysiology of fluent and impaired reading: a magnetoencephalographic approach. J Clin Neurophysiol 17:163-174, 2000

64. Sanai N, Berger MS: Intraoperative stimulation techniques for functional pathway preservation and glioma resection. Neurosurg Focus 28(2):E1, 2010

65. Sanai N, Berger MS: Mapping the horizon: techniques to optimize tumor resection before and during surgery. Clin Neurosurg 55:14-19, 2008

66. Siri S, Tettamanti M, Cappa SF, Della Rosa P, Saccuman C, Scifo P, et al: The neural substrate of naming events: effects of processing demands but not of grammatical class. Cereb Cortex 18:171-177, 2008

67. Sollmann N, Hauck T, Hapfelmeier A, Meyer B, Ringel F, Krieg SM: Intra- and interobserver variability of language mapping by navigated transcranial magnetic brain stimulation. BMC Neurosci 14:150, 2013

68. Sollmann N, Picht T, Mäkelä JP, Meyer B, Ringel F, Krieg SM: Navigated transcranial magnetic stimulation for preoperative language mapping in a patient with a left frontoopercular glioblastoma. J Neurosurg 118:175-179, 2013

69. Sparing R, Mottaghy FM, Hungs M, Brügmann M, Foltys H, Huber W, et al: Repetitive transcranial magnetic stimulation effects on language function depend on the stimulation parameters. J Clin Neurophysiol 18:326-330, 2001

70. Stippich C: [Presurgical functional magnetic resonance imaging.] Radiologe 50:110-122, 2010 (Ger)

71. Stummer W, Reulen HJ, Meinel T, Pichlmeier U, Schumacher W, Tonn JC, et al: Extent of resection and survival in glioblastoma multiforme: identification of and adjustment for bias. Neurosurgery 62:564-576, 2008

72. Suess O, Suess S, Mularski S, Kühn B, Picht T, Schönherr S, et al: [Evaluation of a DC pulsed magnetic tracking system in neurosurgical navigation: technique, accuracies, and influencing factors.] Biomed Tech (Berl) 52:223-233, 2007 (Ger)

73. Szelényi A, Bello L, Duffau H, Fava E, Feigl GC, Galanda $\mathrm{M}$, et al: Intraoperative electrical stimulation in awake craniotomy: methodological aspects of current practice. Neurosurg Focus 28(2):E7, 2010

74. Talacchi A, Santini B, Casagrande F, Alessandrini F, Zoccatelli G, Squintani GM: Awake surgery between art and science. Part I: clinical and operative settings. Funct Neurol 28:205-221, 2013

75. Tarapore PE, Findlay AM, Honma SM, Mizuiri D, Houde JF, Berger MS, et al: Language mapping with navigated repetitive TMS: proof of technique and validation. Neuroimage 82:260-272, 2013

76. Tarapore PE, Martino J, Guggisberg AG, Owen J, Honma
SM, Findlay A, et al: Magnetoencephalographic imaging of resting-state functional connectivity predicts postsurgical neurological outcome in brain gliomas. Neurosurgery 71:1012-1022, 2012

77. Tarapore PE, Tate MC, Findlay AM, Honma SM, Mizuiri D, Berger MS, et al: Preoperative multimodal motor mapping: a comparison of magnetoencephalography imaging, navigated transcranial magnetic stimulation, and direct cortical stimulation. J Neurosurg 117:354-362, 2012

78. Vigliocco G, Vinson DP, Druks J, Barber H, Cappa SF: Nouns and verbs in the brain: a review of behavioural, electrophysiological, neuropsychological and imaging studies. Neurosci Biobehav Rev 35:407-426, 2011

79. Walsh V, Rushworth M: A primer of magnetic stimulation as a tool for neuropsychology. Neuropsychologia 37:125-135, 1999

80. Wassermann EM, Blaxton TA, Hoffman EA, Berry CD, Oletsky H, Pascual-Leone A, et al: Repetitive transcranial magnetic stimulation of the dominant hemisphere can disrupt visual naming in temporal lobe epilepsy patients. Neuropsychologia 37:537-544, 1999

81. Wheat KL, Cornelissen PL, Sack AT, Schuhmann T, Goebel $\mathrm{R}$, Blomert L: Charting the functional relevance of Broca's area for visual word recognition and picture naming in Dutch using fMRI-guided TMS. Brain Lang 125:223-230, 2013

82. Yetkin FZ, Mueller WM, Morris GL, McAuliffe TL, Ulmer JL, Cox RW, et al: Functional MR activation correlated with intraoperative cortical mapping. AJNR Am J Neuroradiol 18:1311-1315, 1997

\section{Author Contributions}

Conception and design: Krieg. Acquisition of data: Krieg, Ille, Sollmann, Hauck, Maurer, Obermueller, Negwer, Droese, Zimmer. Analysis and interpretation of data: Krieg, Ille, Tanigawa, Zimmer. Drafting the article: Krieg, Ille. Critically revising the article: Krieg, Sollmann, Obermueller, Negwer, Droese, Zimmer, Meyer, Ringel. Reviewed submitted version of manuscript: Ringel. Approved the final version of the manuscript on behalf of all authors: Krieg. Statistical analysis: Ille, Tanigawa. Administrative/technical/material support: Meyer, Ringel. Study supervision: Krieg, Meyer, Ringel.

\section{Correspondence}

Sandro M. Krieg, Department of Neurosurgery, Klinikum rechts der Isar, Technische Universität München, Ismaninger Str. 22, 81675 Munich, Germany. email: Sandro.Krieg@1rz.tum.de. 